\title{
Hydrothermal Reactions of Pyruvic Acid: Synthesis, Selection, and Self-Assembly of Amphiphilic Molecules
}

\author{
Robert M. Hazen • David W. Deamer
}

Received: 23 June 2006 / Accepted in revised form: 24 August 2006 /

Published online: 29 November 2006

(C) Springer Science + Business Media B.V. 2006

\begin{abstract}
Selection and self-assembly of organic compounds in aqueous phases must have been a primary process leading to emergent molecular complexity and ultimately to the origin of life. Facile reactions of pyruvic acid under hydrothermal conditions produce a complex mixture of larger organic molecules, some of which are amphiphiles that readily self-assemble into cellsized vesicular structures. Chemical characterization of major components of this mixture reveals similarities to the suite of organic compounds present in the Murchison carbonaceous chondrite, some of whose molecules also self-assemble into membranous vesicles. Physical properties of the products are thus relevant to understanding the prebiotic emergence of molecular complexity. These results suggest that a robust family of prebiotic reaction pathways produces similar products over a range of geochemical and astrochemical environments.
\end{abstract}

Keywords pyruvic acid · hydrothermal system $\cdot$ Murchison carbonaceous chondrite self-assembly · amphiphile

\section{Introduction}

The ancient transition from a geochemical to a biochemical world required a sequence of emergent steps, each of which increased the chemical and structural complexity of Earth's near-surface environment. Among these steps must have been the synthesis of a diverse suite of organic molecules, the subsequent selection of useful biomolecules from this complex mixture, and their organization into essential genetic polymers, catalytic enzymes, and encapsulating membranes (Morowitz 2002; Hazen 2005).

R. M. Hazen $(\bowtie)$

Carnegie Institution and NASA Astrobiology Institute,

5251 Broad Branch Road NW, Washington, DC 20015-1305, USA

e-mail: rhazen@gl.ciw.edu

D. W. Deamer

Department of Chemistry and Biochemistry, University of California,

Santa Cruz, CA 95064, USA 
The principal objective of this study was to determine the chemical and physical properties of the end products of reactions that do not proceed spontaneously at ordinary temperatures and pressures, but can be driven by high-pressure/high-temperature regimes in $\mathrm{CO}_{2}$-rich hydrothermal environments. The results of these experiments inform conjectures that hydrothermal systems represent possible sites that contributed to the diversity of prebiotic organic synthesis, while they elucidate the subsequent self-assembly processes required for the emergence of life (Corliss et al. 1981; Wächtershäuser 1988, 1990; Holm 1992; Shock 1992; Shock et al. 1996; Russell and Hall 1997).

\section{Materials and Methods}

We chose pyruvic acid as the primary reactant because of its importance in presumed primitive metabolic pathways (e.g., Wächtershäuser 1988; Morowitz 1992), and because the abiotic synthesis of pyruvic acid under hydrothermal conditions has been demonstrated (Cody et al. 2000). Quench experiments were conducted with reactants sealed in 2.4- or 4.6-mm diameter gold tubes, which were annealed at $900^{\circ} \mathrm{C}$ and washed in boiling hydrochloric acid to remove ferrous metal impurities prior to use. We performed experiments at several pressures $(50$ to $500 \mathrm{MPa})$ and temperatures $\left(150\right.$ to $\left.350^{\circ} \mathrm{C}\right)$ on pyruvic acid (neat), as well as pyruvic acid $+\mathrm{H}_{2} \mathrm{O}$ (in 1:1, 1:10, and 1:100 molar ratios), and pyruvic acid+oxalic acid dihydrate (1:1 molar ratio). Note that oxalic acid dihydrate decomposes at $T>150^{\circ} \mathrm{C}$ to a mixture of $\mathrm{H}_{2} \mathrm{O}+\mathrm{CO}_{2}+\mathrm{H}_{2}$, and thus provides a convenient method to explore effects of volatile chemistry on reaction products. We employed pyruvic acid (Aldrich 98\%), oxalic acid dihydrate (Aldrich $>99 \%$ ), and distilled deionized water in these experiments.

We loaded each gold tube with reactants (15 or $45 \mathrm{mg}$ pyruvate in 2.4- or 4.6- $\mathrm{mm}$ diameter gold tubes, respectively) and then arc-welded the tube while it was half-immersed in liquid $\mathrm{N}_{2}$. Each tube was weighed before welding, after welding, and after being subjected to high-temperature and high-pressure to ensure that no loss of reactants or leakage of products had occurred.

\section{Reaction conditions}

We applied high temperatures and pressures with a gas-media apparatus (Yoder 1950; Cody et al. 2001). During each experiment, the pressurized samples were exposed to temperature ramping at $20^{\circ} \mathrm{C}$ per minute from ambient conditions to within $10^{\circ} \mathrm{C}$ of the target temperature, then at $5^{\circ} \mathrm{C}$ per minute to the desired temperature. We monitored pressure and temperature continuously. At the conclusion of each run, when the furnace power was turned off, the temperature fell $\sim 100^{\circ} \mathrm{C}$ per minute while pressure was maintained. We stored all gold tube reactors in a freezer $\left(T<-5^{\circ} \mathrm{C}\right)$ until they were opened for analysis.

Most of the gold capsules swelled during heating owing to the release of volatiles (predominantly $\mathrm{CO}_{2}$ ). Therefore, we chilled gold tubes in liquid $\mathrm{N}_{2}$ prior to opening, and recorded the weight loss due to the volatile release upon opening (which was frequently explosive). We then dropped each gold tube into a 4-ml vial with Teflon-lined cap for derivatization and product analysis. We pre-cleaned all vials by heating in air at $500^{\circ} \mathrm{C}$ for $8 \mathrm{~h}$. When amphiphilic components were to be investigated, we dropped the tubes into $2.0 \mathrm{ml}$ chloroform (Aldrich ACS Reagent grade, $>99.8 \%$ ) to dissolve such compounds. 
Analytical techniques

We employed gas chromatography-mass spectrometry (GCMS) to analyze experimental products. Approximately $1 \mu \mathrm{gm}$ of pentadecane (Aldrich, $>99 \%$ ) was added to each sample vial as a concentration standard, and organic acids were converted to their volatile propyl esters with a solution of $14 \%$ (volume) $\mathrm{BF}_{3}$-propanol complex in propanol at $90^{\circ} \mathrm{C}$ with standard esterification procedures (Blau and King 1977). We analyzed the esterified reaction products with a Hewlett-Packard 6890 Series Gas Chromatograph interfaced with a 5972 Series quadrupole mass spectrometer. Product concentrations were calculated from the mass spectrometer total-ion current using calibration curves based on response factors of pure standards. In the case of compounds unavailable commercially, we assumed response factors to be equal to those of similar standards. We performed chromatography with a $14 \%$ cyanopropyl $/ 86 \%$ dimethyl silicone capillary column, the stationary phase chosen for its ability to separate olefinic isomers. Identification of unknown compounds relied on the interpretation of fragmentation behavior with electron-impact and chemicalionization mass spectrometry; the latter method employed either methane or isobutane as the ionization gas.

We further characterized these products with surface enhanced laser desorption/ ionization time-of-flight mass spectrometry (SELDI), for which we employed a Ciphergen Series PBS II ProteinChip Reader with Ciphergen H4 chips. Droplets of the chloroformsoluble fraction of pyruvic acid products were allowed to absorb and dry on the H4 chips, and these spots were washed with phosphate-buffered saline solution $(\mathrm{pH}=7.4)$. We employed alpha-cyano-4-hydroxy cinnamic acid (CHCA) as the energy absorbing molecule and $150 \mathrm{~mJ}$ as the maximum laser intensity. We optimized the system for 100 to 25,000 Da, with a maximum mass of 60,000 Da.

Two-dimensional thin layer chromotography was carried out by spotting $50 \mu \mathrm{l}$ aliquots of the chloroform-soluble components onto $20 \times 20 \mathrm{~cm}$ aluminum-backed silicic acid plates (Alltech Inc.) The plates were developed in the first dimension using $n$-hexane-diethylether $4: 1$, then in the second dimension with chloroform. Fluorescent components that separated on the plate were visualized by activating fluorescence with $365 \mathrm{~nm}$ UV light and photographing the plate. The resulting images were compared to fluorescent components extracted from the Murchison carbonaceous meteorite, using the same 2-D chromatographic procedure (Deamer and Pashley 1989).

We investigated surface properties of reaction products with a Kibron Microtrough X instrument. Aliquots of the chloroform-soluble products were dried and the non-volatile components were weighed to determine mass per volume. Aliquots of the extract were spread on $10 \mathrm{mM}$ sulfuric acid. Force/area isotherms were measured by the Wilhelmy method, with units expressed as millinewton per meter ( $Y$-axis) and area per milligram of chloroform-soluble products ( $X$-axis).

\section{Microscopy}

We dried aliquots of the chloroform extract $(20 \mu \mathrm{l}, \sim 60 \mu \mathrm{g})$ on a glass microscope slide to form spots several millimeters in diameter. A $10-\mu 1$ drop of $10 \mathrm{mM}$ phosphate buffer, $\mathrm{pH} 8.5$, was placed in the center of an 18-mm glass cover slip, which was lowered onto the slide so that the drop came into contact with the center of the dried material, then spread to the edges of the cover slip. The process of hydration and self-assembly was observed with a Zeiss Axiovert 200 microscope, using epifluorescence optics (450 nm excitation filter, 
$520 \mathrm{~nm}$ emission filter). We took micrographs over a period of $30 \mathrm{~min}$ following addition of buffer, during which time amphiphilic components self-assembled into vesicular structures.

\section{Results}

Pyruvic acid ( \pm oxalic acid dihydrate or \pm water) reacts readily at all pressures and temperatures studied. For example, in 2-h experiments more than $65 \%$ of pyruvic acid is consumed at $150^{\circ} \mathrm{C}$, whereas $99 \%$ of pyruvic acid is consumed at $250^{\circ} \mathrm{C}$. GCMS reveals an increasing diversity of reaction products with both temperature and pressure. A typical GCMS chromatogram (Figure 1) displays peaks characteristic of specific product molecules that are superimposed at higher temperature and pressure on a broad unresolved complex mixture (UCM) feature that is produced by a complex suite of thousands of different molecular species. We tentatively identify some sharp peaks as oxygen-substituted alkyl aromatics based on their retention times and characteristic ion fragmentation (Table I), but unambiguous identification of specific isomers is difficult. General analysis of ion fragmentation patterns points to several characteristic functional groups, notably benzene rings.

Several significant trends became apparent when reaction products were analyzed. Notably, three dominant reaction pathways consumed pyruvic acid.

1. Decarboxylation to form acetic acid: In all experiments with pyruvic acid in an aqueous solution under hydrothermal conditions, we observed the expected decarboxylation reaction to acetic acid (Brown 1951; Boyer 1973):

$$
\mathrm{C}_{3} \mathrm{H}_{4} \mathrm{O}_{3}+\mathrm{H}_{2} \mathrm{O} \rightarrow \mathrm{C}_{2} \mathrm{H}_{4} \mathrm{O}_{2}+\mathrm{CO}_{2}+\mathrm{H}_{2} .
$$

2. Dimerization and subsequent decarboxylation to form methylsuccinic acid: In many experiments, particularly those at $T>200^{\circ} \mathrm{C}$ and $P<100 \mathrm{MPa}$, the most abundant single molecular product (apart from acetic acid) is the dicarboxylic acid, methysuccinic acid $\left(\mathrm{C}_{5} \mathrm{H}_{8} \mathrm{O}_{4}\right)$. Yields exceed $10 \%$ of total carbon in some experiments:

$$
2\left(\mathrm{C}_{3} \mathrm{H}_{4} \mathrm{O}_{3}\right) \rightarrow \mathrm{C}_{5} \mathrm{H}_{8} \mathrm{O}_{4}+\mathrm{CO}_{2} .
$$

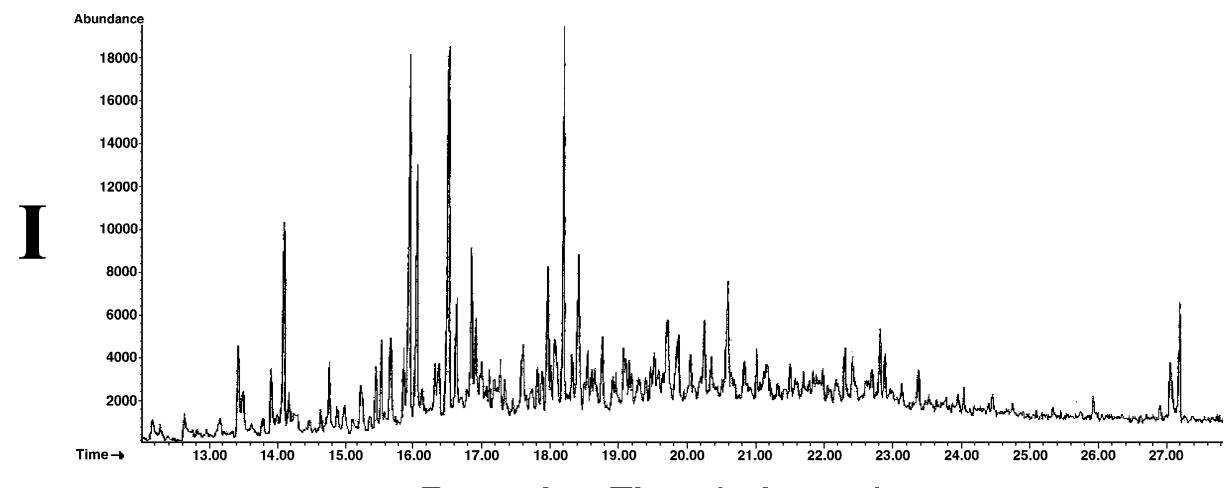

\section{Retention Time (minutes)}

Figure 1 Representative chromatogram of hydrothermal reaction products of pyruvic acid + oxalic acid dihydrate $\left(1: 1\right.$ molar ratio) at $250^{\circ} \mathrm{C}$ and $200 \mathrm{GPa}(2 \mathrm{~h})$. 
TABLE I Possible products from hydrothermal reactions of pyruvic acid at $250^{\circ} \mathrm{C}$ and $0.2 \mathrm{GPa}$, tentatively identified by gas chromatography and characteristic ion fragmentation

\begin{tabular}{ll}
\hline Product & Principal ion fragments \\
\hline Benzene, 1, 2, 3-trimethoxy-5-methyl- & $53,65,79,107,124,139,167,182$ \\
Benzene, 1- (1,3-dimethyl-3-butenyl)-3-methoxy- & $65,77,91,105,135$ \\
Benzene, 1-methoxy-4-(1-methylethyl)- & $51,65,77,91,105,135$ \\
Benzene, 1, 2-diethyl-3, 4, 5, 6-tetramethyl- & $160,175,190$ \\
Benzene, 1, 2, 3-trimethoxy-5-(2-propenyl)- & $77,91,105,133,177,193,208$ \\
1,2-benzenediol, 4- (1, 1-dimethylethyl)- & $77,91,123,151,166$ \\
Benzoic acid, 3-methoxy-, methyl ester & $77,92,107,135,166$ \\
Benzoic acid, 3, 5-dimethyl-, methyl ester & $77,105,133,164$ \\
Benzoic acid, 2, 4, 5-trimethyl-, methyl ester & $77,91,119,147,178$ \\
1,2-benzodioxole-5-carboxylic acid, methyl ester & $63,121,149,180$ \\
1,4-benzenediol, 2-methoxy- & $55,69,97,125,140$ \\
1,4-benzenediol, 2- (1, 1-dimethylethyl)- & $55,123,151,166$ \\
1,4-benzenedicarboxylic acid, dimethyl ester & $76,103,135,163,194$ \\
2-Cyclopenten-1-one, 2, 3, 4-trimethyl- & $53,67,81,96,109,124$ \\
3-cyclopentene-1-carboxylic acid, 3, 4-dimethyl-, methyl ester & $55,67,79,94,95,123,139,154$ \\
1-methoxy-1, 3-cyclohexadiene & $67,79,81,95,110$ \\
Thymol (phenol, 5-methyl-2-(1-methylethyl)- & $65,77,91,105,115,135,150$
\end{tabular}

3. Polymerization and cycloaddition: Condensation polymerization, predominantly to pyruvate dimers and trimers but including longer oligomers, results in a diverse suite of molecules. Some of these oligomers also underwent Diels-Alder cycloaddition (Carey and Sundberg 1993) to form a complex suite of compounds, some of which are amphiphilic. A significant fraction of each capsule's product is soluble in the chloroform phase, representing $>10 \mathrm{wt} \%$ yield based on the original pyruvic acid present. At higher temperatures and pressures more than $20 \%$ of pyruvic acid reacts to yield a water-insoluble, yellow-brown, fluorescent fraction - physical properties that suggest the presence of conjugated bonds. This suite of compounds accounts for the broad UCM feature in gas chromatograms (Figure 1). While details of the complex chromatographs differ for different run compositions ( \pm oxalic acid dehydrate or \pm water) and run conditions (pressure and temperature), we observe qualitatively similar results for all experiments.

A significant fraction of the pyruvic acid product was water insoluble and formed cloudy dispersions in aqueous solutions. This behavior suggests a degree of molecular selection and self-organization typical of lipid-like amphiphilic molecules. Similar behavior was observed in organic compounds extracted from the Murchison carbonaceous chondrite (Deamer and Pashley 1989), as well as in suites of organic molecules synthesized by UV photolysis of interstellar ice analogs in a vacuum chamber (Dworkin et al. 2001). These and other authors (Morowitz et al. 1988; Luisi and Varela 1989; Morowitz 1992; Dobson et al. 2000; Segre et al. 2001; Deamer et al. 2002) argue that amphiphile synthesis and selfassembly are key steps in the origin of boundary membranes that would be required for the origin of cellular life.

We therefore investigated the pyruvic acid products for lipid-like fractions that might display surface activity and the ability to form vesicular structures. Pyruvic acid products from several gold-tube reactor experiments $\left(250^{\circ} \mathrm{C}, 200 \mathrm{MPa}, 2 \mathrm{~h}\right)$ were extracted in $1.0 \mathrm{ml}$ 
chloroform, combined, and analyzed using two-dimensional thin-layer chromatography procedures (2D-TLC), as described by Deamer and Pashley (1989). When TLC plates were analyzed and visualized by exciting fluorescence with $365 \mathrm{~nm}$ UV light, we observed a pattern of fluorescent regions and a concentration of yellow-brown residue at the origin that is strikingly similar to the 2D-TLC pattern reported for chloroform-soluble residues extracted from the Murchison carbonaceous chondrites meteorite (Figure 2).

SELDI mass spectra of the same chloroform-soluble fraction of pyruvic acid products (Figure 3) revealed homologous series of amphiphilic products, presumably monocarboxylic acids ranging up to approximately 20 carbon atoms in length.

In another series of experiments, we tested the chloroform extract of the pyruvic acid product for surface activity, which is a characteristic property of all amphiphilic molecules. The starting material - pyruvic acid - is water soluble, so if surface activity appeared in the reaction products it would be a strong indication that some of the products contained hydrocarbon chains. This would be quite surprising, because it would mean that the pyruvate not only polymerized to form longer chains but furthermore lost oxygen during the reaction to produce hydrocarbons. Surface activity also provides an indication of chain length of the products. Shorter chain amphiphiles such as hexanoic acid do not form stable monolayers, but instead simply dissolve in the subphase. As chain length increases to approximately 10 carbons, amphiphiles become less water soluble and better able to produce monolayers that exhibit surface activity. When amphiphiles reach 18 carbon atoms in length, such as stearic acid, they produce monolayers that are indefinitely stable at the air-water interface.

In order to measure a surface force/area isotherm of the product, a relatively low $\mathrm{pH}$ $(10 \mathrm{mM} \mathrm{HCl}, \mathrm{pH} 2$ ) was required to protonate the carboxylic acid head groups of the
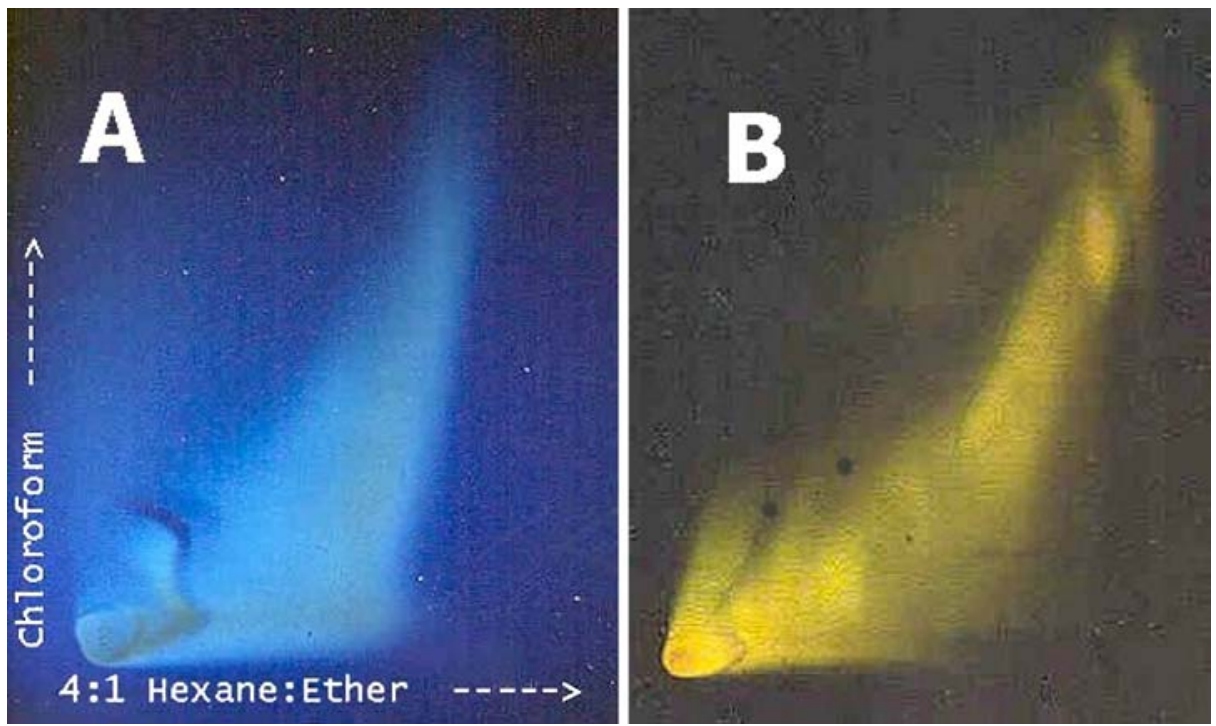

Figure 2 a Distinct fluorescent regions were revealed when components of the chloroform-soluble fraction of pyruvic acid product $\left(250^{\circ} \mathrm{C}, 200 \mathrm{MPa}, 2 \mathrm{~h}\right)$ were separated on a thin-layer silicic acid chromatographic plate. b A thin-layer chromatographic plate for the chloroform-soluble fraction of organic compounds from the Murchison carbonaceous chondrite (Deamer and Pashley 1989) showed distinct similarities to the pyruvic acid product. 


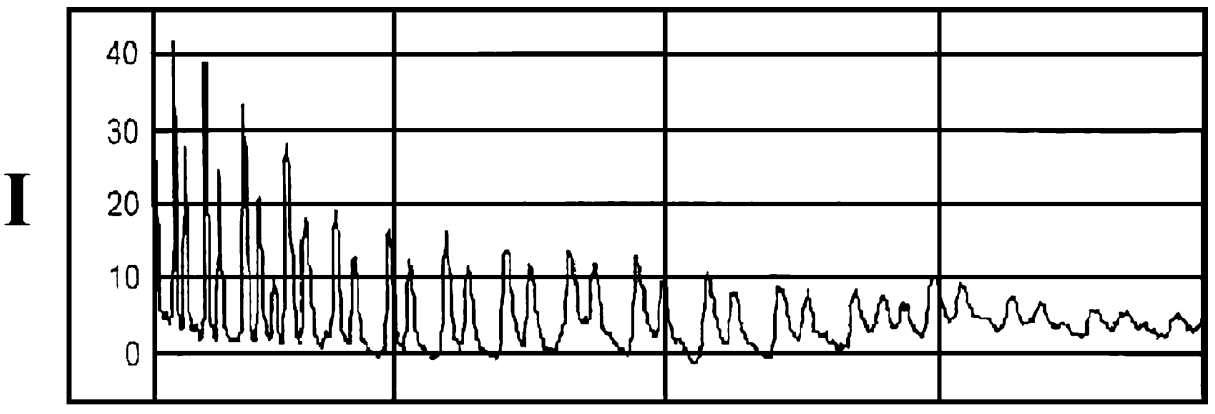

\section{Mass}

Figure 3 A SELDI mass spectrum of the chloroform-soluble fraction of pyruvic acid products revealed a homologous series of amphiphilic products, presumably monocarboxylic acids ranging up to approximately 20 carbon atoms in length. The horizontal scale encompasses masses from $<100$ to $400 \mathrm{Da}$, but the scale is not linear for this time-of-flight technique.

amphiphilic molecules and thereby reduce their solubility in the subphase. We found that surface pressure began to increase at an area per mass of $0.3 \mathrm{~m}^{2} / \mathrm{mg}$, and collapse occurred at $30 \mathrm{mN} \mathrm{m}{ }^{-1}$ when the monolayer area was $0.1 \mathrm{~m}^{2} \mathrm{mg}^{-1}$ (Figure 4). It became clear during such experiments that material was being lost from the monolayer to the subphase. If compression was stopped, the surface pressure decreased over several minutes to an equilibrium spreading pressure of $\sim 6 \mathrm{mN} \mathrm{m}^{-1}$.

At $\mathrm{pH}$ ranges above 7.0, most of the amphiphilic material dissolved in the subphase rather than forming monolayers. This behavior is similar to that previously observed for

Figure 4 The concentration of organic solute $(3.1 \mathrm{mg} / \mathrm{ml})$ was measured by drying a known volume of the chloroform extract and weighing the remaining material. A surface force isotherm was then obtained with a Kilbron instrument as described in the Methods section, using $10 \mathrm{mM}$ sulfuric acid for the subphase.

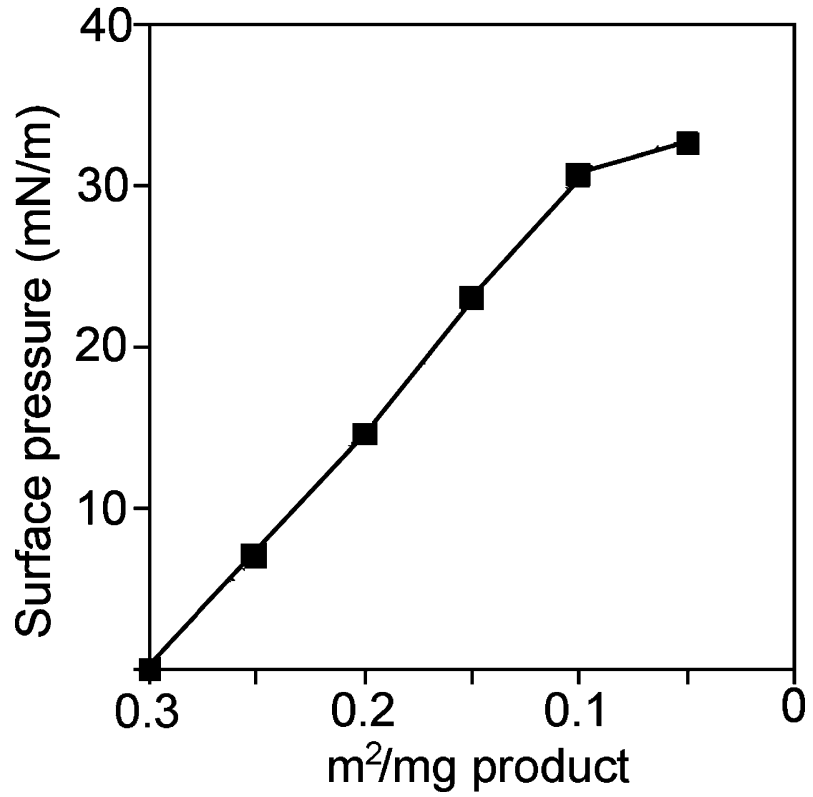


fatty acid monolayers that can dissolve in the subphase (Heikkila et al. 1970). Because the pyruvic acid products include mixtures of monocarboxylic acids ranging from 2 to 18 carbon atoms in length, monolayers would not be expected to be stable, because the shorter chain acids will dissolve in the subphase at varying rates depending on chain length.

The results from the surface force experiments strongly suggested that some of the product components were monocarboxylic acids of sufficient chain length to be surface active and form monolayers at the air-water interface. From previous studies (Hargeaves and Deamer 1978; Walde et al. 1994) we knew that such compounds have the capacity to form membranous vesicles, so we tested samples of the total extract for their ability to undergo self-assembly. The sample was observed by phase and epifluorescence microscopy over a 30-min period as hydration occurred. As expected, virtually none of the sample was soluble in the aqueous phase. Instead, the material slowly dispersed into large numbers of microscopic spherical structures having apparent internal compartments. These vesicular compartments were particularly evident when we examined the structures by epifluorescence microscopy (Figure 5) in which the outer boundaries of individual vesicles were defined by the intrinsic fluorescence of the amphiphilic product.

Figure 5 When a dried aliquot of pyruvic acid products $(\sim 60 \mu \mathrm{g})$ was rehydrated in a phosphatebuffered aqueous solution ( $\mathrm{pH} 8.5)$, vesicles in the size range of 10 to $50 \mu \mathrm{m}$ form spontaneously. The vesicles are autofluorescent. Here the excitation wavelength was filtered at $450 \mathrm{~nm}$, and emitted photons were in the $>500 \mathrm{~nm}$ range.

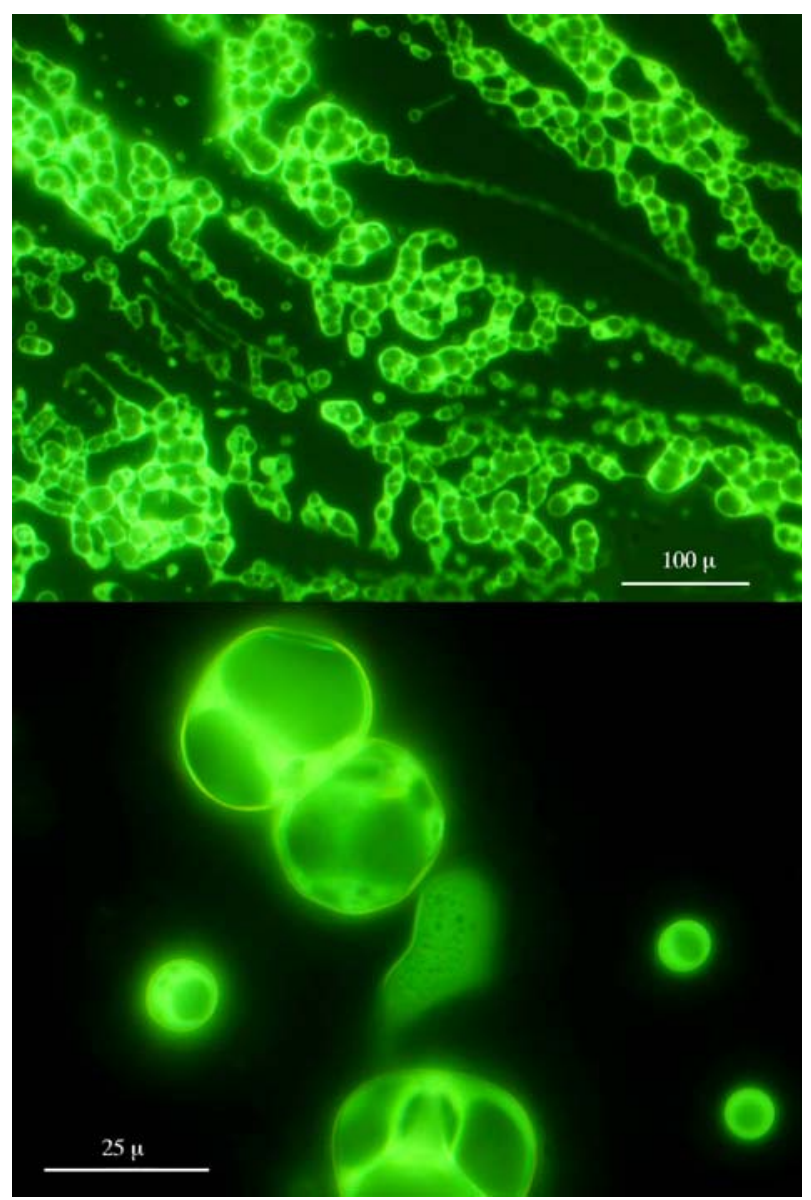




\section{Discussion}

The present experiments on concentrated solutions of pyruvic acid, although conducted at temperatures and pressures appropriate to terrestrial hydrothermal systems, were not intended to mimic prebiotic chemical or physical environments. Rather, we designed these experiments to reveal the nature and extent of reactions that proceed non-enzymatically in relatively concentrated aqueous solutions, and to demonstrate the subsequent self-selection and self-organization of potential prebiotic molecular species from a complex reaction suite. Additional experiments will be required to address whether analogous reactions could occur under plausible geochemical conditions of temperature, pressure, fluid composition, and mineralogical setting.

The hydrothermal experiments described here yield a complex suite of organic molecules which result primarily from the polymerization of pyruvic acid and subsequent reactions, including decarboxylation and cycloaddition. Surprisingly, the products are not a highly cross-linked "tar" as might be expected, but instead the components are soluble in water with a smaller fraction soluble in an organic solvent (chloroform). Some of these bear a strong resemblance to the mixture of chloroform-soluble organic compounds reported by Deamer and Pashley (1989) from the Murchison carbonaceous chondrite. The pattern of distinct fluorescent regions and the concentration near the origin of yellow-brown residuals observed with 2D-TLC are similar in the two samples. Although these two suites of organic species are too complex to analyze in detail, their gross similarities in terms of molecular size, distribution and properties are striking.

Of special note in this study is the synthesis of a significant fraction of amphiphilic species. These are able to promote self-assembly of the vesicular structures shown in Figure 5. However, it should be noted that the membranes are not typical fatty acid bilayers, but instead are thick and viscous, with obvious fluorescent components. Our interpretation is that the membranes are composed of a mixture of relatively non-polar products which are stabilized by surface films of amphiphilic compounds including the monocarboxylic acids indicated by the SELDI trace shown in Figure 3. Further studies are needed to establish the detailed composition and physical properties of the vesicular structures.

Similar reactions in a prebiotic environment would presumably add to the diverse inventory of amphiphilic molecules which spontaneously concentrate and self-organize into membrane-bound enclosures. The only manner in which such products could be synthesized is if pyruvic acid not only polymerized, but also formed hydrocarbon chains of varying length. To account for the properties of surface activity (monolayer formation) and vesicle assembly, the hydrocarbon chains must be at least eight carbon atoms in length. Shorter chain derivatives are too soluble to assemble into stable monolayers and vesicles. The fact that a series of small benzene derivatives are among the common products confirms that dehydration to hydrocarbons can occur. We note that dehydration reactions commonly produce unsaturated bonds in hydrocarbon chains, some of which are likely to be conjugated. If this process occurred during synthesis of pyruvic acid product, it would account for the strong fluorescence we observed.

The similarity of these high-pressure hydrothermal results with those of Deamer and Pashley (1989) for molecules from the Murchison meteorite (presumably formed at lower temperature and pressure ranges), as well as Dworkin et al. (2001) for molecules synthesized in vacuum from UV-irradiated ice, suggests that a robust family of prebiotic reaction pathways may produce similar products over a range of geochemical and astrochemical environments. 
Acknowledgements We gratefully acknowledge the analytical assistance of George Cody (GCMS) and Marilyn Fogel (SELDI). Ren Lu, Berndt Simoniet, Jeremy Sturm, and Mark Teece contributed helpful comments and suggestions. Hatten S. Yoder, Jr. provided invaluable assistance in high-pressure/hightemperature experiments prior to his untimely death in August 2003. This work was supported by grants from NSF Division of Geochemistry (EAR9817964), NASA Exobiology Program (NAGW-4665), NASA Astrobiology Institute (NASA Cooperative Agreement NCC2-1056), and the Carnegie Institution of Washington.

\section{References}

Blau K, King GS (eds) (1977) Handbook of derivatives for chromatography. Heyden, Bellmawr, New Jersey Boyer PD (1973) Carboxylation and decarboxylation. In The enzymes, vol 6, 3rd edn. Academic, New York Brown BR (1951) The mechanism of thermal decarboxylation. Quart Rev 5:131-146

Carey FA, Sundberg RJ (1993) Advanced organic chemistry, part A: structure and mechanisms. Plenum, New York

Cody GD, Boctor NZ, Filley TR, Hazen RM, Scott, JH, Sharma A, Yoder HS Jr (2000) Primordial synthesis of carbonylated iron-sulfur clusters and the synthesis of pyruvate. Science 289:1337-1340

Cody GD, Boctor NZ, Hazen RM, Brandes JA, Morowitz HJ, Yoder HS Jr (2001) Geochemical roots of autotrophic carbon fixation: hydrothermal experiments in the system citric acid- $\mathrm{H}_{2} \mathrm{O}-(\mathrm{FeS})-(\mathrm{NiS})$. Geochem Cosmochim Acta 65:3557-3576

Corliss JB, Baross JA, Hoffman SE (1981) A hypothesis concerning the relationship between submarine hot springs and the origin of life on Earth. Oceanol Acta, Proc 26th Int Geol Congr, Geology of the Oceans Symp., pp. 59-69

Deamer DW, Pashley RM (1989) Amphiphilic components of the Murchison carbonaceous chondrite: surface properties and membrane formation. Orig Life Evol Biosph 19:21-38

Deamer DW, Dworkin JA, Sandford SA, Bernstein MP, Allamandola LJ (2002) The first cell membranes. Astrobiology 2:371-382

Dobson CM, Ellison GB, Tuck AF, Vaida V (2000) Atmospheric aerosols as prebiotic chemical reactors. Proc Natl Acad Sci USA 97:11864-11868

Dworkin JP, Deamer DW, Sanford SA, Allamandola LJ (2001) Self-assembling amphiphilic molecules: synthesis in simulated interstellar/precometary ices. Proc Natl Acad Sci USA 98:815-819

Hargreaves WR, Deamer DW (1978). Liposomes from ionic, single-chain amphiphiles. Biochemistry $17: 3759-3768$

Hazen RM (2005) Genesis: the scientific quest for life's origin. Joseph Henry, Washington, District of Columbia

Heikkila RE, Deamer DW, Cornwell DG (1970) Solution of fatty acids from monolayers spread at the airwater interface: identification of phase transformations and the estimation of surface charge. J Lipid Res $11: 195-200$

Holm NG (ed) (1992) Marine hydrothermal systems and the origin of life. Kluwer, Dordrecht, Netherlands.

Luisi PL, Varela FJ (1989) Self-replicating micelles: a chemical version of a minimal autopoietic system. Orig Life Evol Biosph 19:633-643

Morowitz HJ (1992) Beginnings of cellular life. Yale University Press, New Haven, Connecticut

Morowitz HJ (2002) The emergence of everything. Oxford University Press, New York

Morowitz HJ, Heinz B, Deamer DW (1988) The chemical logic of a minimum protocell. Orig Life Evol Biosph 18:281-287

Russell MJ, Hall AJ (1997) The emergence of life from iron monsulfide bubbles at a submarine hydrothermal redox and $\mathrm{pH}$ front. J Geol Soc 154:377-402

Segre D, Deamer DW, Lancet D (2001) The lipid world. Orig Life Evol Biosph 31:119-145

Shock EL (1992) Chemical environments of submarine hydrothermal systems. Orig Life Evol Biosph 22:67-107

Shock EL, McCollum T, Schulte MD (1996) Geochemical constraints on chemolithoautotrophic reactions in hydrothermal systems. Orig Life Evol Biosph 25:141-159

Wächtershäuser G (1988a) Before enzymes and templates: theory of surface metabolism. Microbiol Rev 52:452-484

Wächtershäuser G (1990) Evolution of the first metabolic cycle. Proc Natl Acad Sci USA 87:200-204

Walde P, Wick R, Fresta M, Mangone A, Luisi PL (1994) Autopoietic self-reproduction of fatty acid vesicles. J Am Chem Soc 116:11649-11654

Yoder HS Jr (1950) High-low quartz inversion up to 10,000 bars. Trans Am Geophys Union 31:821-835 